NSF-ITP-94-28

NUC-MINN-94/3-T

April 1994

\title{
THE PROXIMAL CHIRAL PHASE TRANSITION
}

\author{
Joseph I. Kapusta \\ School of Physics and Astronomy, University of Minnesota, Minneapolis, MN 55455 \\ Ajit M. Srivastava \\ Institute for Theoretical Physics, University of California, Santa Barbara, CA 93106
}

\begin{abstract}
We consider the form of the chiral symmetry breaking piece of the effective potential in the linear sigma model. Surprisingly, it allows for a second local minimum at both zero and finite temperature. Even though chiral symmetry is not exact, and therefore is not restored in a true phase transition at finite temperature, this second minimum can nevertheless mimic many of the effects of a first order phase transition. We derive a lower limit on the height of the second minimum relative to the global minimum based on cosmological considerations; this limit is so weak as to be practically nonexistent. In high energy nuclear collisions, it may lead to observable effects in Bose-Einstein interferometry due to domain walls and to coherent pion emission.
\end{abstract}




\section{Introduction}

The possibility of producing quark-gluon plasma in relativistic heavy ion collisions is an exciting one, especially from the point of view of observing the chiral/confinement phase transition/crossover as the plasma expands and cools. Formation of domains in a chiral phase transition where the chiral field may not be oriented along the true vacuum has been a subject of many investigations recently. Formation of a large domain with a Disoriented Chiral Condensate (DCC) has been proposed by Bjorken, Kowalski and Taylor [1] in the context of high multiplicity hadronic collisions. It was argued in [1] that, as the chiral field relaxes to the true vacuum in such a domain, it may lead to coherent emission of pions [2]. A motivation for this proposal comes from Centauro events in cosmic ray collisions [3]. In the context of quark-gluon plasma, Rajagopal and Wilczek proposed 海 that the nonequilibrium dynamics during the phase transition may produce DCC domains. They argued that long wavelength pion modes may get amplified leading to emission of coherent pions.

One difficulty in these scenarios is that one typically expects domains which are not much bigger than the pion size [5]. Several studies have focussed on the possibility of getting a larger domain. Gavin, Gocksch and Pisarski have argued [5] that large domains of DCC can arise if the effective masses of mesons are small, while Gavin and Müller propose [6] the annealing of smaller domains to give a large region of DCC.

In this paper we consider the structure of the effective potential in the chiral model and note that more general possibilities exist for the symmetry breaking term than considered in these previous investigations. This allows for the existence of a second local minimum of the potential, in addition to the true global minimum, leading to the formation of domain walls which interpolate between the two minima. These walls are unstable unless the two minima are exactly degenerate. We consider constraints coming from cosmology on the parameters responsible for the existence of such walls. We find that such constraints are extremely weak. We then consider

phenomenological consequences of the richer structure of the effective potential of the model, especially from the point of view of the formation of DCC. We show 
that in these more general models large domain walls naturally form but eventually disappear, leading to emission of pions from shell-like structures. Bose-Einstein interferometry should be able to reveal any such shells [7]. We further argue that large regions of DCC may arise naturally in these models, and may be able to account for phenomena like Centauro events.

There is some analogy to the physics of spin glasses [3]. A spin glass is characterized by a phase space which has a complicated landscape of valleys. As the temperature is reduced, barriers between valleys become significant, and the relaxation times become very long. The system may even get trapped in a single metastable state for the duration of the experiment. One may observe hysteresis in strong magnetic fields that varies with the experimental conditions. Real spin glasses have an anisotropy. In hydrodynamic models of Heisenberg spin glasses one is naturally led to consider a coarse-grained free energy which has similarities to the effective potential given in eq. (20) below.

The paper is organized as follows. In sect. 2 we consider the details of the effective potential, both at zero temperature and at finite temperature, and discuss various possibilities for the symmetry breaking term. In sect. 3 we consider constraints on the parameters of the model arising from the formation of domain walls in the early Universe. In sect. 4 we consider potentially observable effects in high energy collisions, namely, pion production from domain walls. In sect. 5 we present some conclusions.

\section{The Effective Potential}

The sigma model [9] is thought to represent the long wavelength limit of QCD [10, 11. Much has been written about the model, both at zero temperature 112, 13] and at finite temperature [14]. Despite this, the shape of the effective potential at both zero and finite temperature may have a nontrivial structure when the pion mass is nonzero, which feature seems to have escaped attention; at least it goes against the conventional considerations. In this section we explore the effective potential, and in the following sections we shall explore its phenomenological consequences. 


\subsection{The vacuum}

We use the sigma model in its linear representation. The Lagrangian is expressed in terms of a scalar field $\sigma$ and the pion field $\boldsymbol{\pi}$.

$$
\mathcal{L}=\frac{1}{2}\left(\partial_{\mu} \sigma\right)^{2}+\frac{1}{2}\left(\partial_{\mu} \boldsymbol{\pi}\right)^{2}-\frac{\lambda}{4}\left(\sigma^{2}+\boldsymbol{\pi}^{2}-c^{2} / \lambda\right)^{2}-V_{S B}
$$

The piece of the Lagrangian which explicitly breaks chiral symmetry is $V_{S B}$. In the absence of this term, the potential has the shape of the bottom of a wine bottle. Chiral symmetry is spontaneously broken in the vacuum, the pion is the massless Goldstone boson, and the $\sigma$ meson gets a mass on the order of $1-2 \mathrm{GeV}$. The axial vector current, defined by

$$
\mathbf{A}_{\mu}=\left(\partial_{\mu} \sigma\right) \boldsymbol{\pi}-\left(\partial_{\mu} \boldsymbol{\pi}\right) \sigma
$$

is conserved. It is believed that chiral symmetry is restored by a phase transition, which is perhaps of second order, at a critical temperature $T_{c} \approx 160 \mathrm{MeV}$; we come back to this point later.

Any quantitative description of Nature at finite momentum and energy must include the vector mesons [15]. Since we are proposing here a qualitatively new phenomenon we shall neglect them, as well as strangeness.

The up and down quarks, while very light, are not massless; therefore, neither is the pion. Historically, there have been three ways to add a symmetry breaking term to the linear sigma model [16]:

1: $V_{S B}=-f_{\pi} m_{\pi}^{2} \sigma$, which is linear in the $\sigma$ field. The PCAC relation

$$
\partial_{\mu} \mathbf{A}^{\mu}=f_{\pi} m_{\pi}^{2} \boldsymbol{\pi}
$$

is realized as an operator equation.

2: $V_{S B}=\frac{1}{2} m_{\pi}^{2} \pi^{2}$, which is quadratic in the pion field. The PCAC relation is realized only in the weak field limit.

3: $V_{S B}=$ constant $\times \bar{N} N$, which is quadratic in the nucleon field. The divergence of the axial vector current is proportional to the axial vector nucleon current.

In principle one could imagine that low energy pion scattering measurements could distinguish between (1) and (2). However, by its very nature that occurs in 
the weak field limit, so the measurements would have to be very precise. Also, low energy pion scattering is influenced by the tails of resonances, such as the $\rho$ meson [17], which would tend to mask the effects of the nonlinear symmetry breaking terms. We are not aware of any experimental evidence which prefers one symmetry breaking term over the other. In this paper we shall not consider the possibility $\mathbf{3}$.

If one insists on an effective Lagrangian which is rotationally invariant and renormalizable, then the most general symmetry breaking potential can be written as

$$
V_{S B}=-\sum_{n=1}^{4} \frac{\epsilon_{n}}{n !} \sigma^{n}+\left(\delta_{1} \sigma+\delta_{2} \sigma^{2}\right) \boldsymbol{\pi}^{2} .
$$

Other symmetry breaking terms one might think of adding simply amount to a redefinition of the 8 parameters $\lambda, c, \epsilon_{n}, \delta_{n}$. Relaxation of the renormalizability condition would allow further terms but, as we shall see, there is already sufficient freedom to generate interesting physics. In what follows we will set $\delta_{1}=\delta_{2}=0$, mainly for simplicity of presentation.

With the symmetry breaking potential as given above, the divergence of the axial vector current is

$$
\partial_{\mu} \mathbf{A}^{\mu}=-V_{S B}^{\prime}(\sigma) \boldsymbol{\pi}
$$

where the prime denotes differentiation with respect to $\sigma$. It is clear that PCAC is an operator identity only if $V_{S B}$ is linear in $\sigma$. The ground state of this theory ought to occur at $\sigma(x)=\sigma_{\mathrm{gs}}>0$ and $\boldsymbol{\pi}(x)=\mathbf{0}$. We must immediately impose three conditions; that the minimum of the full potential occur at $\sigma_{\mathrm{gs}}$, that PCAC hold for small fluctuations about $\sigma_{\mathrm{gs}}$, and that the pion has its physical mass there. Therefore,

$$
\begin{gathered}
V_{S B}^{\prime}\left(\sigma_{\mathrm{gs}}\right)=-f_{\pi} m_{\pi}^{2}, \\
\sigma_{\mathrm{gs}}\left(\lambda \sigma_{\mathrm{gs}}^{2}-c^{2}\right)+V_{S B}^{\prime}\left(\sigma_{\mathrm{gs}}\right)=0, \\
\lambda \sigma_{\mathrm{gs}}^{2}-c^{2}=m_{\pi}^{2},
\end{gathered}
$$

and so $\sigma_{\mathrm{gs}}=f_{\pi}$.

One must still ensure that the global minimum is really obtained when $\boldsymbol{\pi}(x)=$ 0. To investigate this problem, let us expand the fields about an arbitrary point as 
follows.

$$
\begin{aligned}
\sigma(x) & =v \cos \theta+\sigma^{\prime}(x), \\
\boldsymbol{\pi}(x) & =\mathbf{v} \sin \theta+\boldsymbol{\pi}^{\prime}(x) .
\end{aligned}
$$

The primes denote fluctuations about the given point. The full potential is

$$
V(v, \theta)=\frac{\lambda}{4}\left(v^{2}-c^{2} / \lambda\right)^{2}+V_{S B}(v \cos \theta) .
$$

We now consider several limits for the symmetry breaking piece of the potential.

Suppose that $\epsilon_{1}=f_{\pi} m_{\pi}^{2}$ and all other $\epsilon$ 's are zero. Minimizing the potential with respect to $v$ at fixed $\theta$ gives

$$
v(\theta) \approx f_{\pi}(1+\Delta)
$$

where $\Delta=-2\left(m_{\pi} / m_{\sigma}\right)^{2} \sin ^{2}(\theta / 2), m_{\sigma}^{2}=2 \lambda f_{\pi}^{2}+m_{\pi}^{2}$, and

$$
V_{\min }(\theta)-V_{\min }(0) \approx 2 f_{\pi}^{2} m_{\pi}^{2} \sin ^{2}(\theta / 2)
$$

Corrections to the approximate equalities are of relative order $\Delta<<1$. This is a tilted wine bottle bottom with only one minimum.

Now suppose that $\epsilon_{2}=m_{\pi}^{2}$ and all other $\epsilon$ 's are zero. Then

$$
v^{2}(\theta)=f_{\pi}^{2}\left[1-2 m_{\pi}^{2} \sin ^{2} \theta / m_{\sigma}^{2}\right]
$$

where $m_{\sigma}^{2}=2 \lambda f_{\pi}^{2}$, and

$$
V_{\min }(\theta)-V_{\min }(0) \approx \frac{1}{2} f_{\pi}^{2} m_{\pi}^{2} \sin ^{2} \theta
$$

We neglect a correction of relative order $\left(m_{\pi} / m_{\sigma}\right)^{2}$. This potential has two degenerate minima located at $\theta=0$ and $\pi$ !

In general, one may expect that $V_{S B}$ allows for two minima, one at $\theta=0$ and one at $\theta=\pi$. If they are not degenerate then by a simple redefinition of the fields we may, by convention, choose $\theta=0$ to be the global minimum. We shall investigate what limits cosmology may place on the existence and depth of the second minimum in the next section; we shall find that the constraint is extremely weak. It is quite surprising to us that neither terrestrial experiments nor pure 
theoretical computations in QCD so far tell us anything about a possible second minimum.

Our statement certainly goes against the conventional point of view as expounded in ref. [18], for example, which says that the symmetry breaking potential should be linear in the fields, and for three quark flavors should follow the $\left(3,3^{*}\right)+\left(3^{*}, 3\right)$ symmetry breaking scheme of Gell-Mann, Oakes and Renner [19]. The main argument seems to be that this is the simplest description which gives reasonable low energy phenomenology. There was some interest in this issue in the 1970's in regard to three flavor physics. It was found that the addition of symmetry breaking terms bilinear in the scalar fields resulted in low energy phenomenology as good as, or better than, linear terms alone [20]. These bilinear terms could have the structure $\left(3,3^{*}\right)+\left(3^{*}, 3\right)$ or they could have components of some other group structure. A more recent study [21] has found a nonzero coefficient of a bilinear term in the three flavor nonlinear sigma model. We will not pursue the three flavor world in this paper, but it is certainly worth doing.

One might at first think that $\epsilon_{n} \propto m_{q}^{n}$ (where $m_{q}$ is the up or down quark mass) so that $\epsilon_{1}$ is much greater than $\epsilon_{2}$ and so on. We think it is quite possible that all $\epsilon_{n} \propto m_{q}$ and therefore of comparable magnitude (when scaled appropriately with $\left.f_{\pi}\right)$. The argument is that the sigma model is only a low energy effective model of QCD and all possible terms which are allowed should be included. Indeed, even if one started originally with only a linear symmetry breaking term $\epsilon_{1}^{\prime}$, loop corrections would generate nonlinear terms. These nonlinear terms would have coefficients equal to $\epsilon_{1}^{\prime}$ times some function of $\lambda$ and $c$. Since $\lambda$ and $c$ are big we expect that all $\epsilon_{n} f_{\pi}^{n}$ would turn out to be comparable in magnitude.

For clarity of exposition we shall hereafter restrict our attention to the possibility that only $\epsilon_{1}$ and $\epsilon_{2}$ are nonzero. This is sufficient to parametrize the effective potential with the freedom to adjust the tilt of the bottom of the wine bottle as well as the depth of the second minimum. We have then at our disposal four parameters in the effective Lagrangian: $\lambda, c, \epsilon_{1}$ and $\epsilon_{2}$. These parameters must be restricted so as to give the proper pion mass, pion decay constant, a reasonable value for the $\sigma$ mass, PCAC in the weak field limit, and the condition that the ground state of the 
theory occur at $\sigma=f_{\pi}$ and $\boldsymbol{\pi}=\mathbf{0}$. We obtain

$$
\begin{aligned}
m_{\pi}^{2} & =\lambda f_{\pi}^{2}-c^{2}, \\
m_{\sigma}^{2} & =2 \lambda f_{\pi}^{2}+m_{\pi}^{2}-\epsilon_{2}, \\
f_{\pi} m_{\pi}^{2} & =\epsilon_{1}+\epsilon_{2} f_{\pi} .
\end{aligned}
$$

The numerical values chosen in this paper are $m_{\pi}=140 \mathrm{MeV}$ and $f_{\pi}=94.5 \mathrm{MeV}$. Lin and Serot 22] have emphasized that the $\sigma$ meson in this model is not to be identified with the exchange of two correlated pions in the isoscalar - scalar channel in the nucleon - nucleon interaction. That exchange is rather broadly distributed in mass with a peak around $600 \mathrm{MeV}$. Good phenomenology for low energy pion and nucleon dynamics is obtained if the $\sigma$ meson has a mass greater than about $1 \mathrm{GeV}$. For definiteness, we choose $m_{\sigma}=1 \mathrm{GeV}$. We shall vary $\epsilon_{1}$ between 0 and $f_{\pi} m_{\pi}^{2}$. There is no further freedom given the above constraints.

\section{$2.2 \quad$ Finite temperature}

To estimate what may happen at finite temperature we will calculate thermal fluctuations to one loop order and furthermore take the high temperature limit. Quantitatively this cannot be very accurate. The relevant coupling constant is large: $\lambda \approx\left(m_{\sigma} / f_{\pi}\right)^{2} / 2 \approx 50$. Qualitatively the result should be alright; however, see 田, 23].

To proceed, we expand the fields about an arbitrary point, as in eqs. (9-10), and determine the masses of the fluctuations. If $\mathbf{v}$ points in the third direction in isospin space then the eigenvalues of the mass-squared matrix are

$$
\begin{aligned}
& m_{1}^{2}=\lambda v^{2}-c^{2} \\
& m_{2}^{2}=\lambda v^{2}-c^{2} \\
& m_{3}^{2}=2 \lambda v^{2}-c^{2}-\frac{\epsilon_{2}}{2}-\sqrt{\lambda^{2} v^{4}-\epsilon_{2} \lambda v^{2} \cos (2 \theta)+\frac{\epsilon_{2}^{2}}{4}} \\
& m_{0}^{2}=2 \lambda v^{2}-c^{2}-\frac{\epsilon_{2}}{2}+\sqrt{\lambda^{2} v^{4}-\epsilon_{2} \lambda v^{2} \cos (2 \theta)+\frac{\epsilon_{2}^{2}}{4}} .
\end{aligned}
$$

In the high temperature limit of the one loop approximation one keeps only the terms of order $T^{4}$ and $m^{2} T^{2}$. Ignoring terms which are independent of $v$ and $\theta$ we 
get the simple expression

$$
V(v, \theta ; T)=\frac{\lambda}{4} v^{4}-\frac{1}{2}\left(c^{2}+\epsilon_{2} \cos ^{2} \theta-\frac{\lambda T^{2}}{2}\right) v^{2}-\epsilon_{1} v \cos \theta .
$$

In the chiral limit one finds, as is well-known, a second order phase transition at the critical temperature $T_{c}=\sqrt{2 c^{2} / \lambda}=\sqrt{2} f_{\pi}$. An analysis by Karsch [24] of all available lattice simulations of two-flavor QCD extrapolated to zero quark mass is consistent with a second order transition with critical indices the same as the $\mathrm{O}(4)$ model. So at least qualitatively the model and approximations made here make sense. However, as emphasized by Shuryak [25], the sigma model is supposed to represent only the long wavelength modes, and certainly does not include the contribution from short wavelength modes. For example, as one approaches $T_{c}$ from below, the model does not include the $\eta, \rho, \omega$, and the whole tower of mesons above them. As one approaches $T_{c}$ from above, the model does not include all the degrees of freedom represented by quarks and gluons. The energy density of the long wavelength modes represented by the pion and sigma degrees of freedom should be thought of as sitting on top of a much larger energy density represented by all these other degrees of freedom.

When the up and down quark masses are nonzero chiral symmetry is not exact. It cannot be restored at high temperature. If $V_{S B}$ is an even function of $\sigma$ the Lagrangian still possesses a discrete symmetry which is restored at some critical temperature. For example, when only $\epsilon_{2} \neq 0$, this temperature is $\sqrt{2\left(c^{2}+m_{\pi}^{2}\right) / \lambda}$.

It is straightforward to show that the zero temperature effective potential has a second, local, minimum at $\theta=\pi$ which is separated from the global minimum at $\theta=0$ by a barrier when the inequality $\epsilon_{2} \sqrt{c^{2} / \lambda}>\epsilon_{1}>0$ is satisfied. $\left[\sqrt{c^{2} / \lambda}\right.$ is just $f_{\pi}$ up to corrections of order $m_{\pi}^{2} / m_{\sigma}^{2}$.] As the temperature is increased, this minimum develops into a saddle at temperature $T_{1}$ where the curvature in the azimuthal direction changes from positive to negative. The saddle eventually disappears at the higher temperature $T_{2}$. Here

$$
T_{1}=\sqrt{2\left[\frac{c^{2}}{\lambda}-\left(\frac{\epsilon_{1}}{\epsilon_{2}}\right)^{2}\right]},
$$


and

$$
T_{2}=\sqrt{\frac{2\left(c^{2}+\epsilon_{2}\right)}{\lambda}-6\left(\frac{\epsilon_{1}}{2 \lambda}\right)^{2 / 3}} .
$$

In figures 1 through 4 we show the evolution of the effective potential with increasing temperature for four sets of $\epsilon$. All of these represent a slice through the $V-\sigma$ plane, and are normalized such that $V_{\min }=0$ at each temperature. One can imagine that a system cooling through $T_{1}$ could get trapped in the metastable minimum. We shall consider such possibilities in the following sections. We call this the proximal chiral phase transition, since it is a consequence of the proximity of exact chiral symmetry in parameter (quark mass) space, but it is not a true phase transition in the thermodynamic sense.

\section{Cosmological Constraints}

Considerations of phase transitions in the early Universe have been very useful in restricting particle theory models. We now ask whether cosmology places any constraints on the parameters characterizing the effective potential in eq. (1). As mentioned earlier, we will be considering only $\epsilon_{1}$ and $\epsilon_{2}$ to be non-zero as this is sufficient to capture the qualitative aspects of the effective potential. It is wellknown that when the effective potential has more than one disconnected minima then domain walls are produced in a phase transition. For the potential term in eq. (4) this happens if $\epsilon_{1}=0$ and $\epsilon_{2} \neq 0$. Two regions of space which correspond to the two degenerate vacua $\sigma=f_{\pi}$ and $\sigma=-f_{\pi}$, respectively, (see eq. (14)), will be separated by a domain wall where the chiral field smoothly interpolates between the two vacua. In the context of the early Universe, stable domain walls are almost always disastrous unless the phase transition happens at extremely late times. This already suggests that the parameter $\epsilon_{1}$ cannot be identically zero.

By expanding $\sigma$ and $\boldsymbol{\pi}$, as in eqs. (9-10), we can determine the effective Lagrangian for $\theta$ from eqs. (1) and (20) to be

$$
\mathcal{L}_{\theta}=\frac{v^{2}}{2}\left(\partial_{\mu} \theta\right)^{2}+\epsilon_{1} v \cos \theta+\frac{\epsilon_{2}}{2} v^{2} \cos ^{2} \theta .
$$

Here $v$ minimizes the effective potential at fixed $\theta$ and $T$; that is, it traces the 
bottom of the valley of the potential. Since this valley has an almost constant radius at fixed $T$, we can neglect its very weak dependence on $\theta$. Hence $v=v(T)$.

As we will show in the following, cosmology places a lower limit on $\epsilon_{1}$ which is very small. Thus, as far as cosmological considerations are concerned, we can determine the structure of the domain wall by taking $\epsilon_{1}=0$. With this, and defining $\theta^{\prime} \equiv 2 \theta$, we get the following equation of motion for $\theta^{\prime}$,

$$
\square \theta^{\prime}+\epsilon_{2} \sin \theta^{\prime}=0
$$

This is the familiar sine-Gordan equation which is known to have the domain-wall solutions 26

$$
\theta^{\prime}(z)=4 \tan ^{-1}\left[\exp \left(\sqrt{\epsilon}_{2} z\right)\right]
$$

where the $z$ axis is normal to the wall. The thickness of the wall is $\delta \simeq \epsilon_{2}^{-1 / 2}$. Thus the surface energy density $\rho_{S}$ of the wall is of the order

$$
\rho_{S} \simeq \epsilon_{2} v^{2} \delta=v^{2} \sqrt{\epsilon}_{2} .
$$

When $\epsilon_{1}$ is nonzero then, even though domain walls still form, they are not stable any more. [It is simple to check that domain walls always form as long as $\epsilon_{1}<f_{\pi} \epsilon_{2}$, which is just the condition that there be a local minimum at $\theta=\pi$.] Instability of the wall arises because now one minimum $(\theta=0)$ is energetically preferred over the other $(\theta=\pi)$ so the region corresponding to $\theta=\pi$ shrinks while the region corresponding to $\theta=0$ expands. These unstable domain walls then disappear in the course of time as the true minimum spreads throughout space. Of course, in the context of the early Universe, there is an upper bound on the life-time of such walls if they are not to dominate the energy density of the Universe. As the standard theory of nucleosynthesis is in very good agreement with observations, one would also like that any such unstable domain walls do not influence it.

A restriction on the life-time of the unstable walls implies restriction on the parameters $\epsilon_{1}$ and $\epsilon_{2}$, which we now consider. [Other parameters in eq. (1) are not constrained by such considerations from cosmology; the only other topological objects in the model are Skyrmions, which are supposed to be nucleons.] Let us refer to the difference in the energy densities at $\theta=\pi$ and $\theta=0$ as $\Delta \rho_{V}$. From 
eq. (23) we have $\Delta \rho_{V}=2 \epsilon_{1} v$. One expects that the instability of domain walls becomes significant when the age of the Universe $t^{*}$ is such that the energy excess at the scale $t^{*}$ becomes comparable to the energy of the domain wall on the same scale [27].

A good way to understand this is to realize that when $\epsilon_{1} \neq 0$ one is actually considering a situation similar to a first order phase transition. As domain walls keep intersecting each other and pinching off, one can consider closed domain walls at any given time. The whole region then looks like bubbles of one phase embedded in the other other. The bubbles which have $\theta=\pi$ inside will always shrink, while the bubbles which have $\theta=0$ inside should expand, but only if the size of such bubbles is larger than a critical size $R_{c}$. This critical size can be determined by considering the total energy $E_{R}$ of a bubble of radius $R$,

$$
E_{R}=-\frac{4 \pi}{3} R^{3} \Delta \rho_{V}+4 \pi R^{2} \rho_{S} .
$$

The critical size $R_{c}$ is determined by requiring that $E_{R}$ be stationary with respect to small variations in $R$. Bubbles larger than $R_{c}$, which have $\theta=0$ inside, will expand while those smaller than $R_{c}$ will collapse. We find that

$$
R_{c}=\frac{2 \rho_{S}}{\Delta \rho_{V}}=v \frac{\sqrt{\epsilon}_{2}}{\epsilon_{1}}
$$

So far we have neglected the fact that the Universe is expanding. If $R_{c}$, at a given time, comes out larger than the horizon size, at that time, then all the bubbles with sizes smaller than the horizon will shrink. Bubbles with sizes equal to or greater than the horizon always stretch along with the horizon due to the expansion of the Universe, irrespective of which phase is enclosed. Therefore, in order that the difference between the global minimum and the metastable minimum becomes important, $R_{c}$ must be smaller than the horizon size $H$ (corresponding to the age of the Universe at time $t^{*}$ ). Using the above equation, this implies that

$$
H \geq v \frac{\sqrt{\epsilon}_{2}}{\epsilon_{1}}
$$

Using the constraint given by eq. (18) we can write this as

$$
H \geq \frac{v \sqrt{\epsilon_{2}}}{f_{\pi}\left(m_{\pi}^{2}-\epsilon_{2}\right)}=\frac{v \sqrt{m_{\pi}^{2}-\epsilon_{1} / f_{\pi}}}{\epsilon_{1}} .
$$


The horizon size at the time the Universe passed through the chiral/confinement phase transition/crossover is about $10^{6} \mathrm{~cm}$, which is very large compared to QCD scale of $10^{-13} \mathrm{~cm}$. Unless $\epsilon_{2}$ is extremely close to $m_{\pi}^{2}$ the above inequality will be trivially satisfied. [Note that the domain walls do not really have to disappear much before the time of nucleosynthesis. Therefore the real constraint is somewhat weaker than this.] Since $v \leq f_{\pi}$ we find the lower bound to be

$$
\epsilon_{1}>3 \times 10^{-13} \mathrm{MeV}^{3}
$$

As long as $\epsilon_{1}$ is larger than this the domain walls will disappear very quickly and will not affect the Universe in any significant way. Because the horizon is very small at that time, any density fluctuations generated by collapsing domain walls will also get wiped out quickly.

Clearly, the constraint on $\epsilon_{1}$ given by eq. (31) (and the corresponding constraint on $\epsilon_{2}$ ) is extremely weak. Since eq. (1) describes an effective theory anyway, it is safe to say that cosmology imposes no practical constraints on the parameters of this model.

\section{High Energy Nuclear Collisions}

We now consider chiral symmetry breaking in the context of quark-gluon plasma formation in a heavy ion collision and the influence of misaligned chiral condensate on pion production. As we mentioned in the introduction, the possibility of coherent pion emission from extended domains is very interesting and has been a subject of

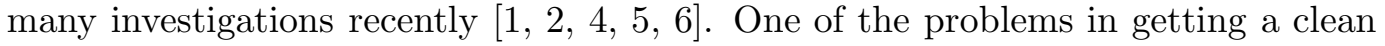
signature is that such domains are expected to be very small [5]. However, these investigations have been restricted to the case when only $\epsilon_{1}$ is non-zero. As we have discussed earlier, there does not seem any reason to exclude other symmetry breaking terms in the potential. The structure of the system drastically changes when we consider $\epsilon_{2}$ also non-zero, as exemplified by the presence of domain walls. In this section we will consider what sorts of signatures one can expect for these more general possibilities for the symmetry breaking terms in the effective potential.

Let us first consider pion production due to different regions of misaligned 
condensate. Since previous studies have considered only $\epsilon_{1}$ non-zero, we first briefly comment on this case. It has a uniformly tilted potential with unique minimum at $\sigma>0$. It has been suggested earlier 海 that, in a rapid phase change, the chiral field could roll down to different minima in different regions. In a given region, a pion condensate could form if the chiral field points in a direction different from the true minimum. As this pion field relaxes to the true minimum it will lead to coherent emission of pions.

One difficulty with getting a clean signal in this scenario is that the typical domain one expects is very small, of order $1 \mathrm{fm}[5]$. However, we would like to point out that this does not exclude the possibility of the formation of pion condensate in a large region. For example, consider two adjacent domains where the chiral field points in two different directions, say $\theta_{1}$ and $\theta_{2}$. As the chiral field relaxes, one may expect that both $\theta_{1}$ and $\theta_{2}$ will approach zero. However, this really depends on the values of these angles. For example, assume that $\theta_{1}=\pi+\alpha_{1}$ and $\theta_{2}=\pi-\alpha_{2}$, where both $\alpha_{1}$ and $\alpha_{2}$ are small. Then in the region where the two domains are in contact, the chiral field will have to smoothly interpolate between the two angles, and hence somewhere in that region it will point in the direction $\pi$. As the outer regions of the domains relax to $\theta=0$, the chiral field may start to cover a larger region of the order parameter space $\mathcal{M}$. [We use $\mathcal{M}$ to denote the manifold defined by the bottom of the valley traced by the minimum of the effective potential.] This means that the evolution of the chiral fields in a collection of domains are not totally uncorrelated. Essentially, the chiral field defines a smooth map from the region covered by the domains into the manifold $\mathcal{M}$. The image of this map is actually a smooth and connected patch in the manifold $\mathcal{M}$. Smoothness of this patch is enforced by the condition that the chiral field must smoothly interpolate in-between any two adjacent domains. When the chiral field relaxes, this patch deforms as its portions slide down to the global minimum. It is quite conceivable that this type of evolution of the chiral field leads to a pion condensate pointing in a direction which is in some way the average direction defined by the patch. It is thus not clear that small individual domains imply no pion condensate in large regions. 
We will not, however, pursue this line of argument since $\epsilon_{2}=0$ is unnecessarily restrictive. Rather, we will show that a non-zero value of $\epsilon_{2}$ leads to the existence of large domains in a very natural manner.

As discussed in sect. 2, non-zero values of $\epsilon_{2}$ lead to the existence of domain walls if the inequality $\epsilon_{1}<f_{\pi} \epsilon_{2}$ is satisfied. [If not then there is no qualitative difference between this case and the case with $\epsilon_{2}=0$.] These walls are unstable for all non-zero values of $\epsilon_{1}$. Consider the chiral phase change in a region of the quark-gluon plasma. As now there are two minima, a local minimum at $\sigma=v(\pi)$ and the global minimum at $\sigma=v(0)$, the chiral field can relax to either of these two values. One therefore expects a domain pattern as shown in figure 5a. For pion production, the initial size of these domains is not crucial to our model; this clearly distinguishes our case from previous considerations, where the size of the initial domain was crucial. In figure 5a we have denoted different domains by the angle to which the chiral field relaxes in that region.

Initially, when the temperature is high, the global minimum is only very slightly preferred over the local minimum. At those early times, small walls may collapse but large ones may simply be stretched by the expansion of the plasma. This is very similar to the situation in the early Universe. As the plasma cools, the energy density decreases, eventually reaching a point when the walls become unstable in the sense that the $\theta=0$ minimum becomes favorable over the $\theta=\pi$ minimum. If the expansion is slow then all the walls with sizes smaller than the critical size [as given by eq. (28)] will shrink and disappear. In any case, one is led to a hierarchy of sizes of collapsing walls (see figure 5b) which will lead to emission of pions. As the walls collapse, the average domain size will increase. Once the typical domain size becomes larger than about $R_{c}$, the instability of walls will become significant. After this the regions with $\theta=0$ will expand and the regions with $\theta=\pi$ will contract.

The simplest prediction is the formation of walls with size of order $R_{c}$ carrying excess energy density. It is important to note that these large walls will form irrespective of the size of the initial domains. These walls may expand, converting the false minimum into the true one, or they may collapse if they enclose the false minimum. One would expect generally that a large wall (comparable to the size of 
the system) may be left enclosing the true minimum as shown in figure 6 . Between this wall and the outer boundary of the plasma region the chiral field is in the metastable phase. As the $\theta=0$ phase expands both from the outside and from the inside the two walls will meet. This will lead to a shell of the size of the system containing high surface energy density. All the energy contained in the two walls will be converted to pions.

The most important feature of these pions is that they ought to be emitted from a shell-like region; studies of Bose-Einstein correlations of pions should be able to reveal such a shell structure. Investigations in [f] could be useful in this context. A second feature is the possibility that the pions emitted from such a shell, or from the collapsing walls, may be coherent. We now address this possibility.

Consider a closed wall which bounds a region of the false minimum embedded in the true minimum. This wall will collapse and all the energy contained in the wall will be converted into pions. The initial structure of the wall is determined by the details of the variation of the chiral field from the $\theta=0$ region to the $\theta=\pi$ region. We remind ourselves that we are actually dealing with the minimum of the effective potential which is topologically a three-sphere $S^{3}$. Different portions of the wall correspond to different trajectories which the chiral field traces from the north pole of this $S^{3}$ to its south pole. Clearly there is no reason to expect that different portions of the wall will all correspond to the same curve on $S^{3}$ initially. One can then think of the initial distribution of the chiral field in the entire wall as a thick strip joining the north and the south poles of $S^{3}$.

As the wall collapses, one will also expect the thickness of this strip to decrease because a thick strip costs gradient energy. Since the pion is very light, the shrinking of a strip on $S^{3}$ may proceed faster than the wall shrinks, especially for the walls which do not shrink initially, either due to the expansion of the plasma, or due to being least unstable. If this strip on $S^{3}$ shrinks significantly before the wall completely collapses, then the entire collapsing shell will correspond to a chiral field which, though still interpolating between $\theta=0$ and $\theta=\pi$, goes through a unique plane. For example, this curve may lie entirely in the $\pi_{1}-\pi_{2}$ plane. This might lead to a pion condensate, culminating in all of the wall energy being emitted in coherent 
pions. [Of course, the process of shrinking of the strip on $S^{3}$ also will produce pions but they will not be coherent.] The same consideration can be applied to the type of situation in figure 6 where coherent pions may be emitted from a shell-like structure.

Let us make a rough estimate of the energy contained in the walls. As an example, take $\epsilon_{2}=0.8 m_{\pi}^{2}$. Then with eqs. (18) and (28) we get the size of the critical bubble to be $R_{c} \simeq 4.5 m_{\pi}^{-1}$. With the surface energy density of the wall as given in eq. (26) the net energy contained in the wall is about $14 \mathrm{GeV}$. This is a very large energy which can lead to a high multiplicity of pions. In this estimate we have only considered the energy of the wall, neglecting the difference in energy of the two minima. During the collapse (or expansion) of the wall, false minimum energy will be converted into the kinetic energy of the wall which should be included to get the net energy emitted in pions.

We briefly discuss the possibility that our model can also account for Centaurolike events [3]. A highly energetic cosmic ray collision may produce a tiny bubble of false vacuum such that the bubble wall propogates initially outwards due to the initial momentum (or may be due to the initial expansion of the partons). Eventually this wall will collapse back. As this wall first expands and then collapses, there may be enough time to develop a pion condensate on the wall (due to shrinking of the strip on $S^{3}$ in the sense described above), and hence lead to the emission of coherent pions. This is especially likely as the initial size of the bubble may be very small so the pion field configuration in its wall may be pretty much uniform any way. Another possibility is that as the bubble of false vacuum expands due to initial wall momentum, a true vacuum bubble of critical size nucleates inside it. This then may lead to a large spherical shell containing high surface enrgy density (similar to that in figure 6). All this energy may then be emitted in coherent pions.

If we assume that all of the coherent pions come from a $\theta=\pi$ bubble, which eventually collapses, then the energy emitted in pions $E_{\pi}$ can be related to the radius $R_{F}$ of this bubble using eq. (27) as

$$
E_{\pi}=4 \pi f_{\pi}\left[f_{\pi} \sqrt{\epsilon_{2}} R_{F}^{2}+\frac{2}{3} \epsilon_{1} R_{F}^{3}\right]
$$

$R_{F}$ here represents the radius of the bubble at its largest size. The entire event will include any hadrons produced when the bubble was nucleated as well as the hadrons 
emitted at the end when the bubble completely collapses. Presumably coherent pions will be emitted at the end of the event. If we assume a relation between $R_{F}$ and the duration of the event $\tau$, say $R_{F} \sim \tau^{a}$, where $a$ is some parameter, then our model predicts a very specific dependence of $E_{\pi}$ on $\tau$ (given by eq. (32)). If the information of $E_{\pi}$ and $\tau$ is experimentally available, then this equation can be fitted with data to check our model and hopefully get the parameters $a, \epsilon_{1}$ and $\epsilon_{2}$.

\section{Conclusion}

Our main idea is that the effective potential, or free energy, resulting from chiral Lagrangian models of QCD may have a second metastable minimum at a chiral angle of $\pi$. We illustrated this in the linear sigma model with two quark flavors. Our numerical examples were restricted to a symmetry breaking potential which had terms linear and quadratic in sigma but, in general, there is no reason to think that the other possible terms are ignorable. There does not seem to be any fundamental reason why one should not take this situation seriously. Indeed, three flavor models with linear plus bilinear terms were investigated briefly in the 1970's. The motivation then was to investigate the pattern of symmetry breaking, the analytic behavior of observables as the symmetry breaking parameters were sent to zero, and to obtain improved phenomenology. It should be kept in mind that the parameters in a two flavor model can be renormalized by the presence of a heavier, third flavor. Generalizations of our study to three flavors should be done.

Cosmology places a constraint on the height of a possible second minimum relative to the true minimum. This constraint arises from the requirement that the energy in domain walls not upset standard calculations of nucleosynthesis. The constraint is so weak that it has no practical consequences for high energy particle or nuclear experiments.

High energy nuclear collisions seem to present a remarkable opportunity to study the topography of the effective chiral potential at finite temperature. Nonzero up and down quark masses spoil the ideal chiral symmetry and smear out the probable second order phase transition. This may be a cloud with a silver lining if a second metastable minimum exists as it could mimic the effects of a first order 
phase transition. We have argued that formation and evolution of domains, with their attendent domain walls, can plausibly lead to observable consequences. These include coherent pion emission and Bose-Einstein interferometry of shell structures. Detailed predictions with a specific effective potential require numerical simulations as well as the inclusion of vector mesons.

\section{Acknowledgements}

This work was begun during the program Strong Interactions at Finite Temperature at the Institute for Theoretical Physics in the fall of 1993. It was supported by the U.S. Department of Energy under grant number DOE/DE-FG02-87ER40328 and by the U.S. National Science Foundation under grant number PHY89-04035. We thank J. D. Bjorken, D. K. Campbell, B. Holstein, R. D. Pisarski, J. Polchinski, J. Schechter and K. Rajagopal for discussions.

\section{References}

[1] J.D. Bjorken, K.L. Kowalski and C.C. Taylor, preprint SLAC-PUB-6109, presented at the 7th Les Rencontres de Physique de la Vallee d'Aoste: Results and Perspectives in Particle Physics, La Thuile, Italy, 7-13 March 1993.

[2] J. P. Blaizot and D. Diakonov, Phys. Lett. B315, 226 (1993).

[3] C. M. G. Lattes, Y. Fujimoto and S. Hasegawa, Phys. Rep. 65, 151 (1980); L. T. Baradzei et al., Nucl. Phys. B370, 365 (1992), and references therein.

[4] K. Rajagopal and F. Wilczek, Nucl. Phys. B399, 395 (1993); Nucl. Phys. B404, 577 (1993).

[5] S. Gavin, A. Gocksch and R. D. Pisarski, Phys. Rev. Lett. 72, 2143 (1994). See also, D. Boyanovsky, H. J de Vega and R. Holman, preprint, University of Pittsburgh, PITT-94-01.

[6] S. Gavin and B. Müller, preprint BNL-GM-1, Dec. 1993.

[7] S. Pratt, Phys. Rev. Lett. 53, 1219 (1984). 
[8] A standard review of spin glasses is: K. Binder and A. P. Young, Rev. Mod. Phys. 58, 801 (1986).

[9] M. Gell-Mann and M. Levy, Nuovo Cimento 16, 705 (1960).

[10] S. Weinberg, Physica 96A, 327 (1979).

[11] J. Gasser and H. Leutwyler, Ann. Phys. (N.Y.) 158, 142 (1984).

[12] B. W. Lee, Chiral Dynamics (Gordon and Breach, New York, 1972).

[13] J. F. Donoghue, E. Golowich and B. R. Holstein, Dynamics of the Standard Model (Cambridge University Press, 1992).

[14] R. Pisarski and F. Wilczek, Phys. Rev. D29, 338 (1984); F. Wilczek, Int. J. Mod. Phys. A7, 3911 (1992).

[15] U.-G. Meissner, Phys. Rep. 161, 213 (1988).

[16] D. K. Campbell, Phys. Rev. C19, 1965 (1979).

[17] J. F. Donoghue, C. Ramirez and G. Valencia, Phys. Rev. D39, 1947 (1989); J. F. Donoghue and B. R. Holstein, Phys. Rev. D40, 2378 (1989).

[18] H. Pagels, Phys. Rep. 16, 219 (1975).

[19] M. Gell-Mann, R. J. Oakes and B. Renner, Phys. Rev. 175, 2195 (1968).

[20] See, for example: D. W. McKay, W. F. Palmer and R. F. Sarraga, Phys. Rev. D8, 2532 (1973); A. A. Khelashvili, Nucl. Phys. B90, 336 (1975); H. B. Geddes and R. H. Graham, Phys. Rev. D13, 56 (1976); A. K. Bhargava, Phys. Rev. D18, 1737 (1978).

[21] J. Schechter, A. Subbaraman and H. Weigel, Phys. Rev. D48, 339 (1993).

[22] W. Lin and B. D. Serot, Phys. Lett. B233, 23 (1989); Nucl. Phys. A512, 637 (1990).

[23] S. Gavin, A. Gocksch and R. D. Pisarski, Phys. Rev. D49, R3079 (1994).

[24] F. Karsch, Phys. Rev. D49, 3791 (1994). 
[25] E. Shuryak, preprint NSF-ITP-93-123 (1993).

[26] A. Vilenkin and A. E. Everett, Phys. Rev. Lett. 48, 1867 (1982).

[27] A. Vilenkin, Phys. Rev. D23, 852 (1981). 


\section{Figure Captions}

Figure 1: Temperature dependence of the effective potential for the choice of parameters $\epsilon_{1}=\epsilon_{2}=0$. The curve shown represents a slice through the $V-\sigma$ plane. The potential is rotationally symmetric. There is a second order phase transition at $T_{c}=\sqrt{2} f_{\pi}=133.6 \mathrm{MeV}$.

Figure 2: Temperature dependence of the effective potential for the choice of parameters $\epsilon_{1}=f_{\pi} m_{\pi}^{2}$ and $\epsilon_{2}=0$. The curve shown represents a slice through the $V-\sigma$ plane. The bottom of the potential is tilted. There is a saddle point at $\theta=\pi$ for $T<114.9 \mathrm{MeV}$.

Figure 3: Temperature dependence of the effective potential for the choice of parameters $\epsilon_{1}=0$ and $\epsilon_{2}=m_{\pi}^{2}$. The curve shown represents a slice through the $V-\sigma$ plane. There is a second order phase transition restoring the discrete symmetry $\sigma \rightarrow-\sigma$ at $T_{c}=\sqrt{2} f_{\pi}=133.6 \mathrm{MeV}$.

Figure 4: Temperature dependence of the effective potential for the choice of param-

eters $\epsilon_{1}=0.25 f_{\pi} m_{\pi}^{2}$ and $\epsilon_{2}=0.75 m_{\pi}^{2}$. The curve shown represents a slice through the $V-\sigma$ plane. The direction $\theta=\pi$ has a local minimum when $T<T_{1}=123.2$ $\mathrm{MeV}$, a saddle point when $T_{1}<T<T_{2}=127.0 \mathrm{MeV}$, and no critical point at all when $T>T_{2}$.

Figure 5: (a) Formation of domains after the phase transition. Domains denoted by 0 and $\pi$ here represent regions where the chiral field has settled to the true and the false minimum, respectively. Solid lines separating different domains show the initial structure of domain walls. Outermost solid line denotes the boundary of the system. (b) As domain walls join and collapse a hierarchy of domain sizes is generated. The solid lines again represent domain walls separating different minima.

Figure 6: Eventually one may be left with a large shell-like domain of metastable matter. As this domain shrinks, one will be left with a large shell (of the size of the system) containing large surface density. 

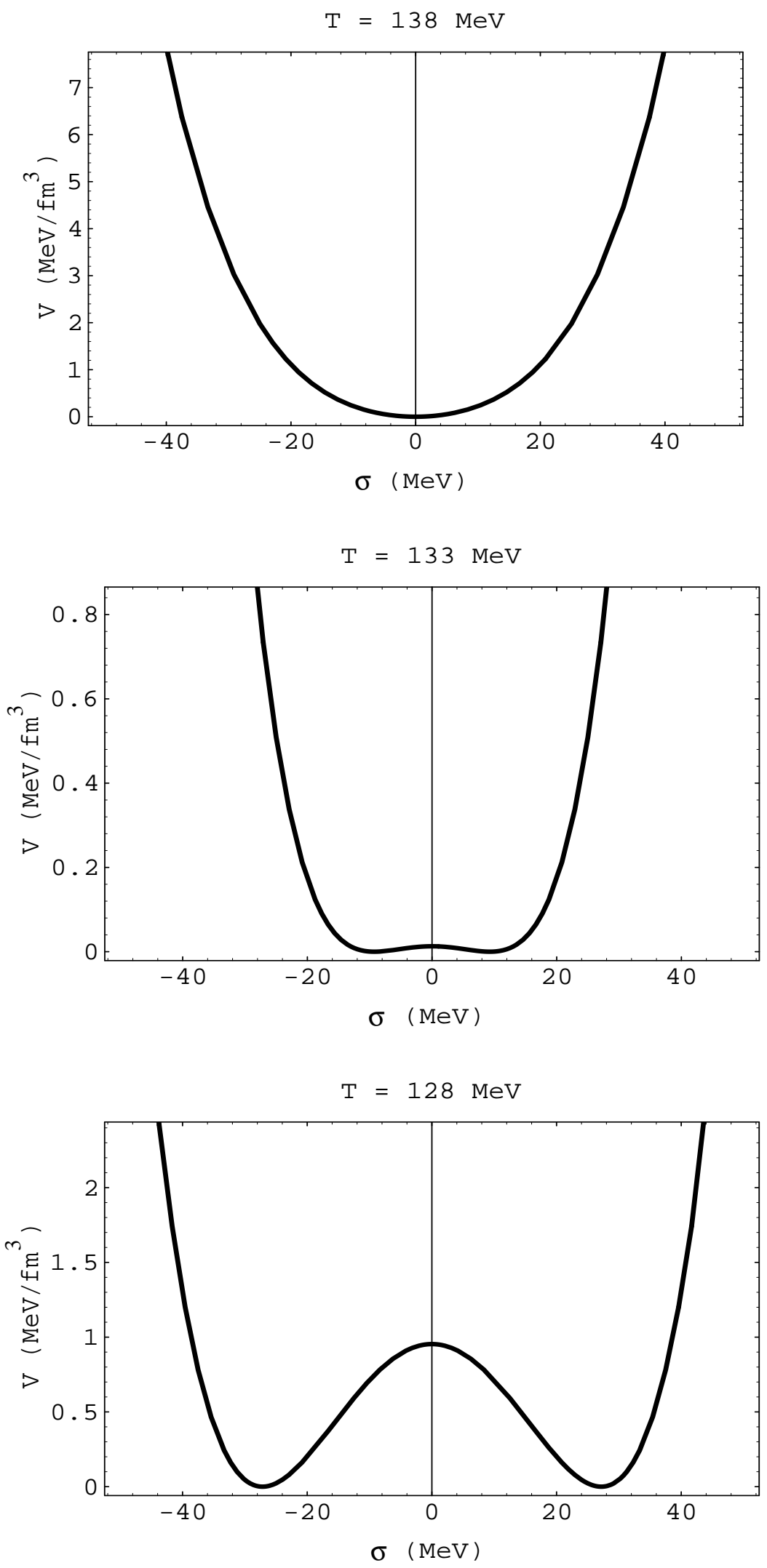

Fig. 1 
This figure "fig1-1.png" is available in "png" format from: http://arxiv.org/ps/hep-ph/9404324v1 
This figure "fig2-1.png" is available in "png" format from: http://arxiv.org/ps/hep-ph/9404324v1 

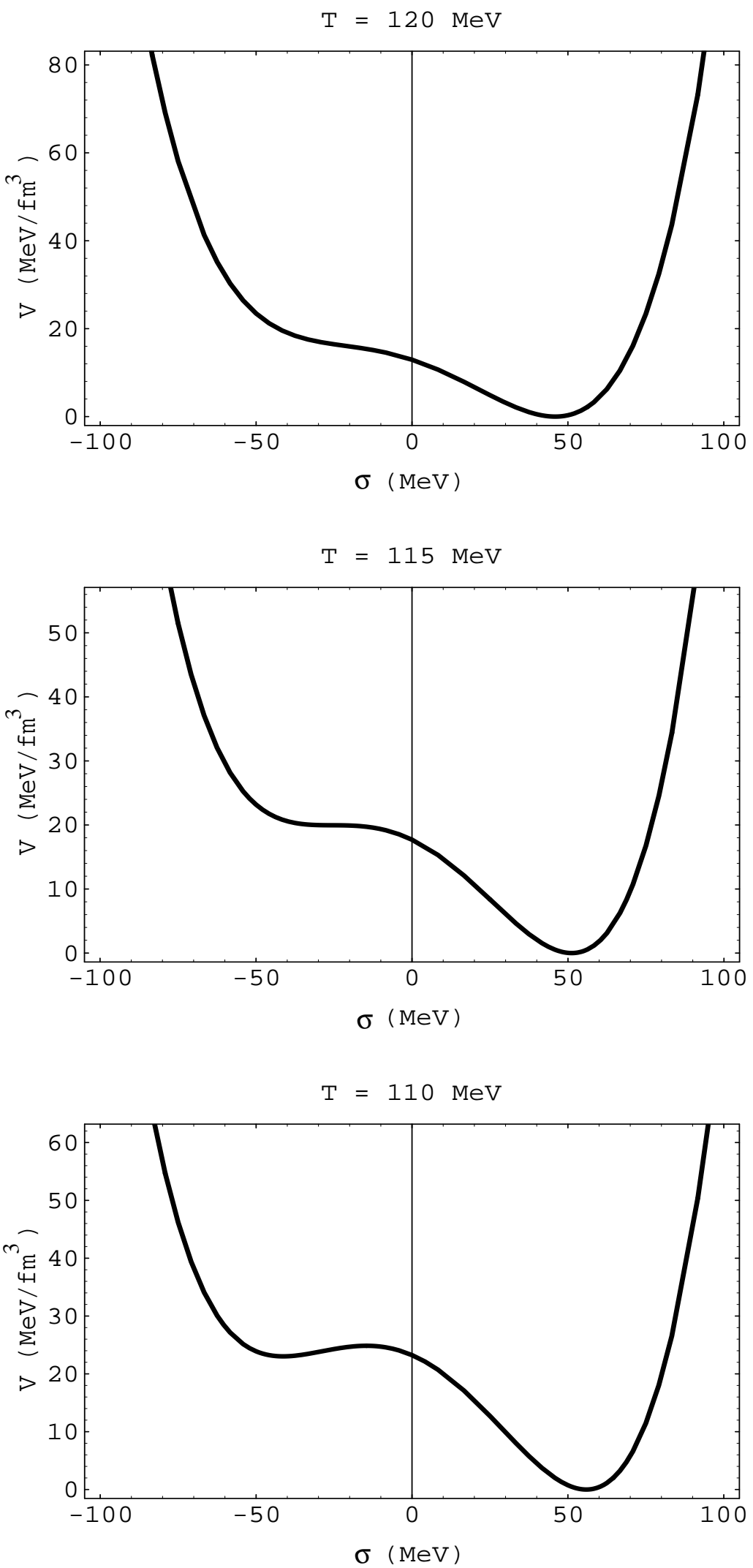

Fig. 2 
This figure "fig1-2.png" is available in "png" format from: http://arxiv.org/ps/hep-ph/9404324v1 
This figure "fig2-2.png" is available in "png" format from: http://arxiv.org/ps/hep-ph/9404324v1 

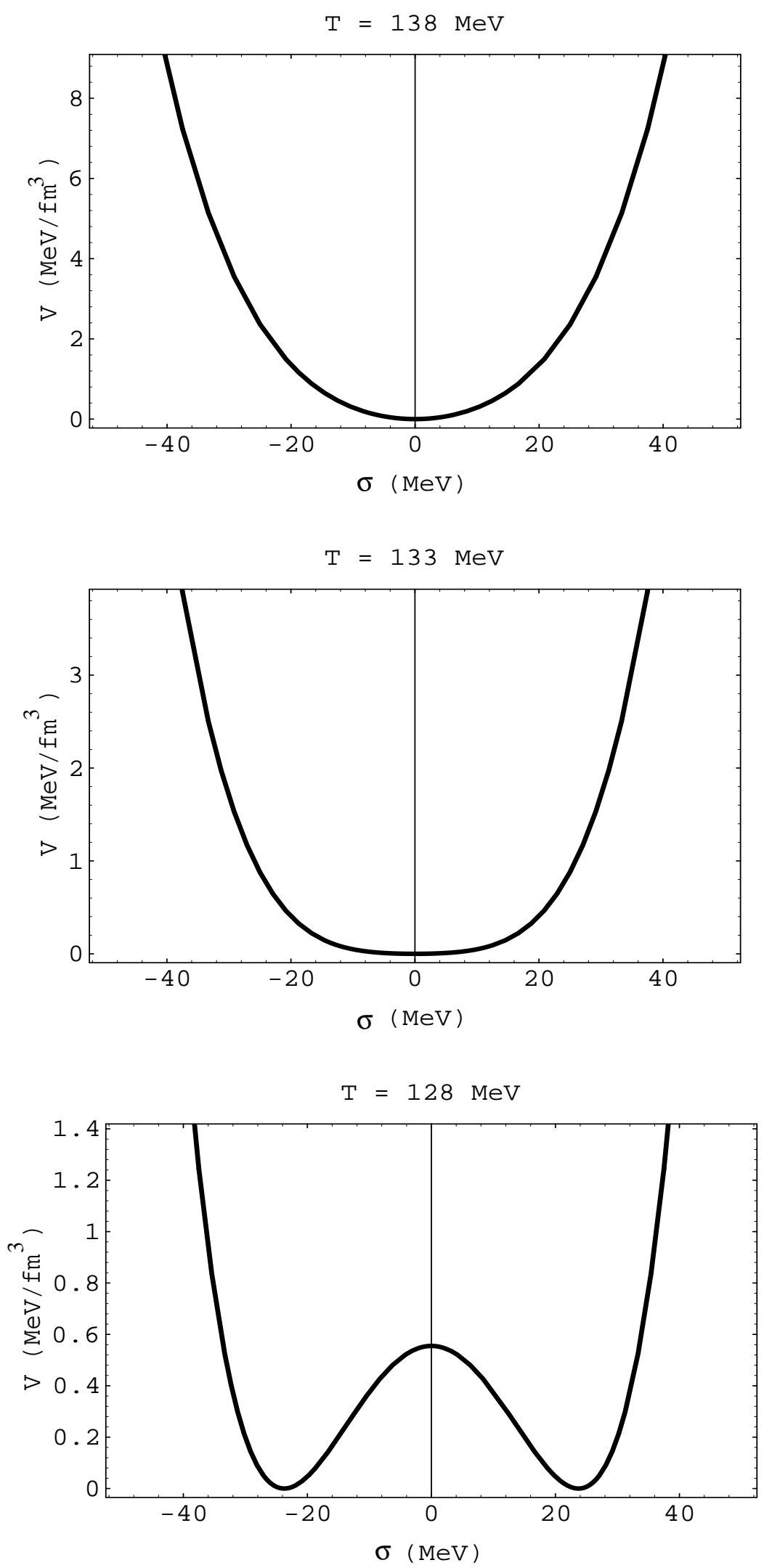

Fig. 3 
This figure "fig1-3.png" is available in "png" format from: http://arxiv.org/ps/hep-ph/9404324v1 

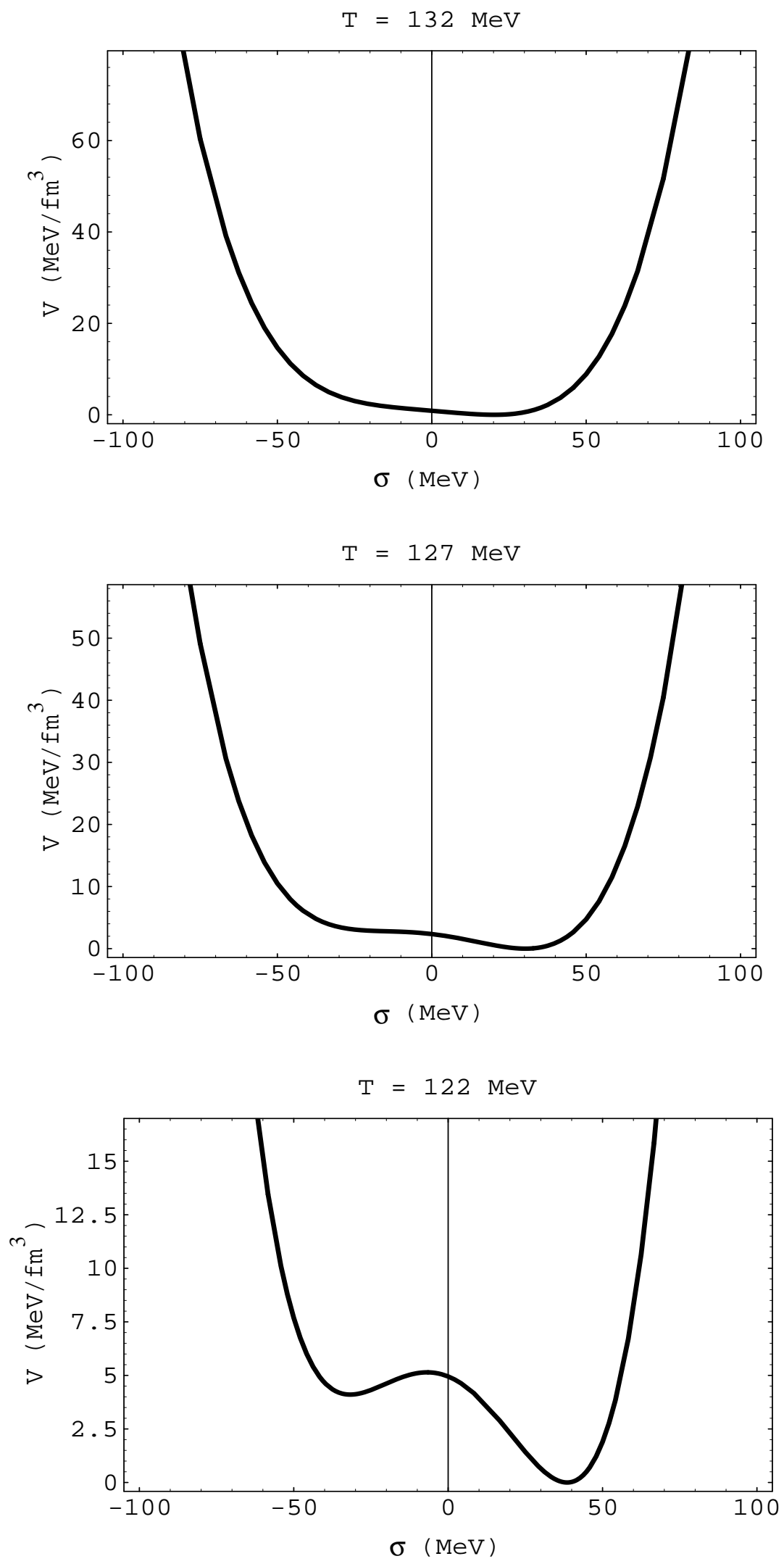

Fig. 4 
This figure "fig1-4.png" is available in "png" format from: http://arxiv.org/ps/hep-ph/9404324v1 


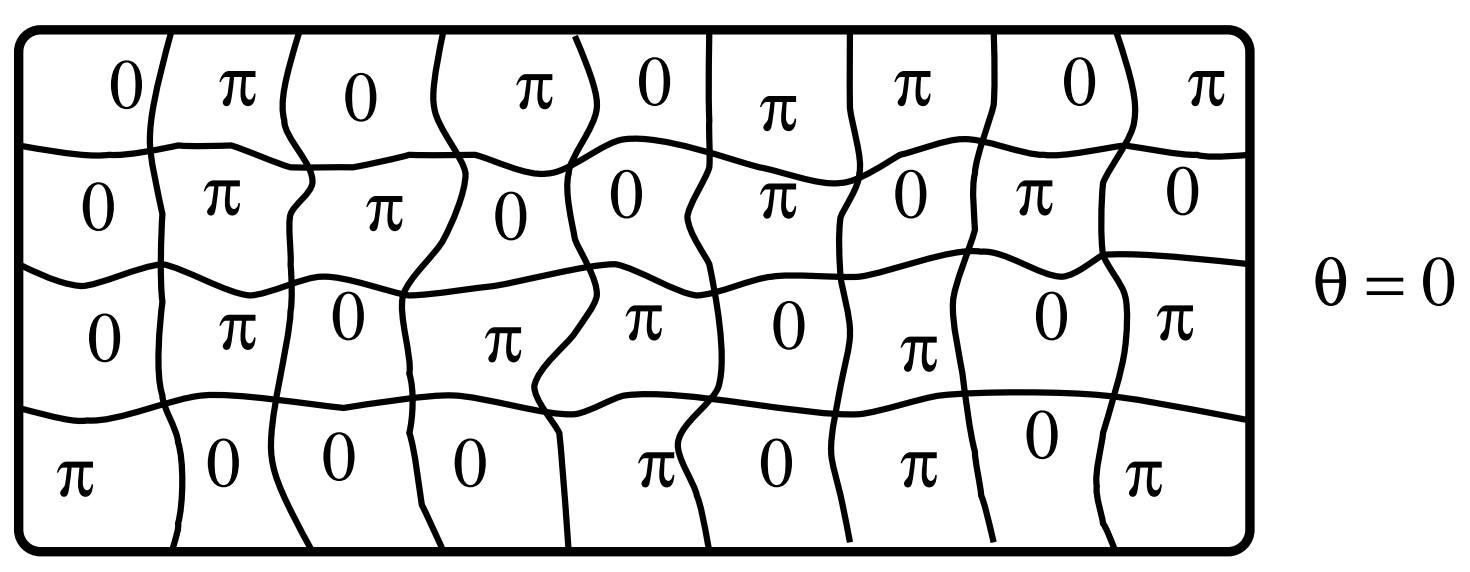

(a)

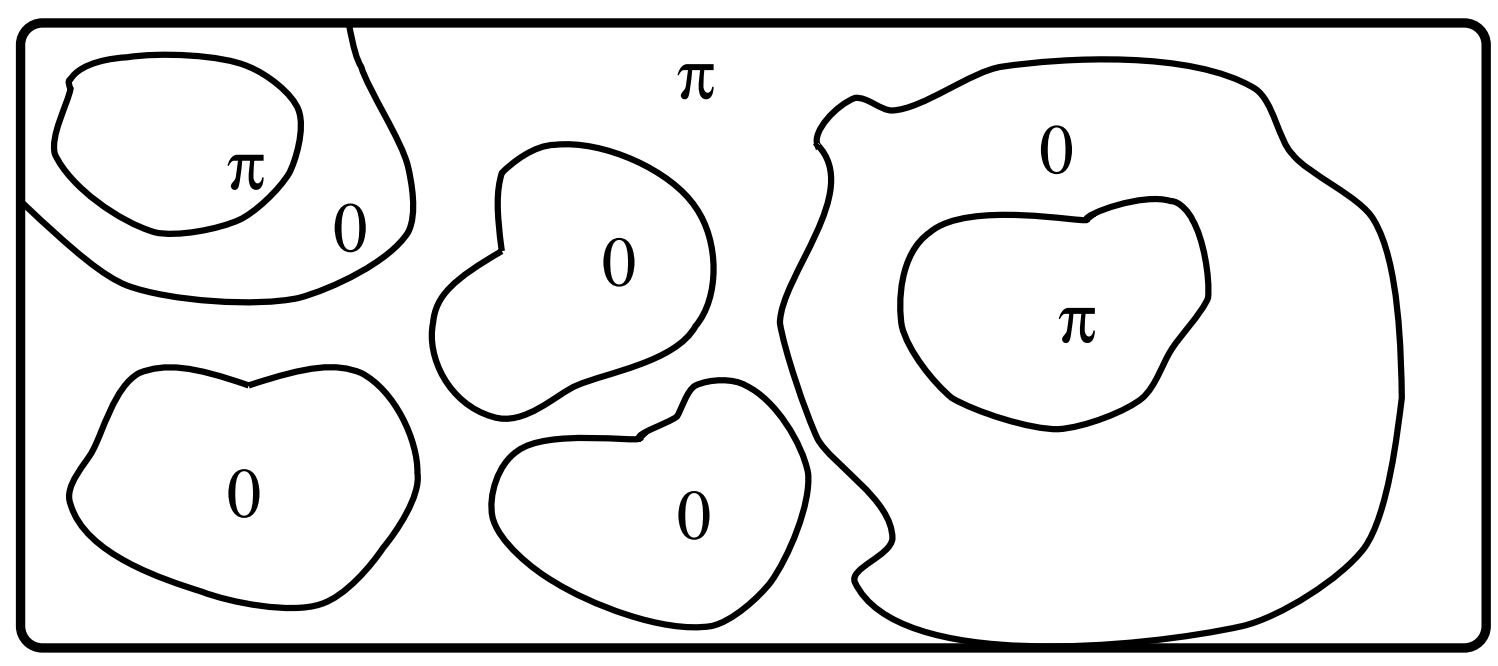

$\theta=0$

(b)

Fig. 5 


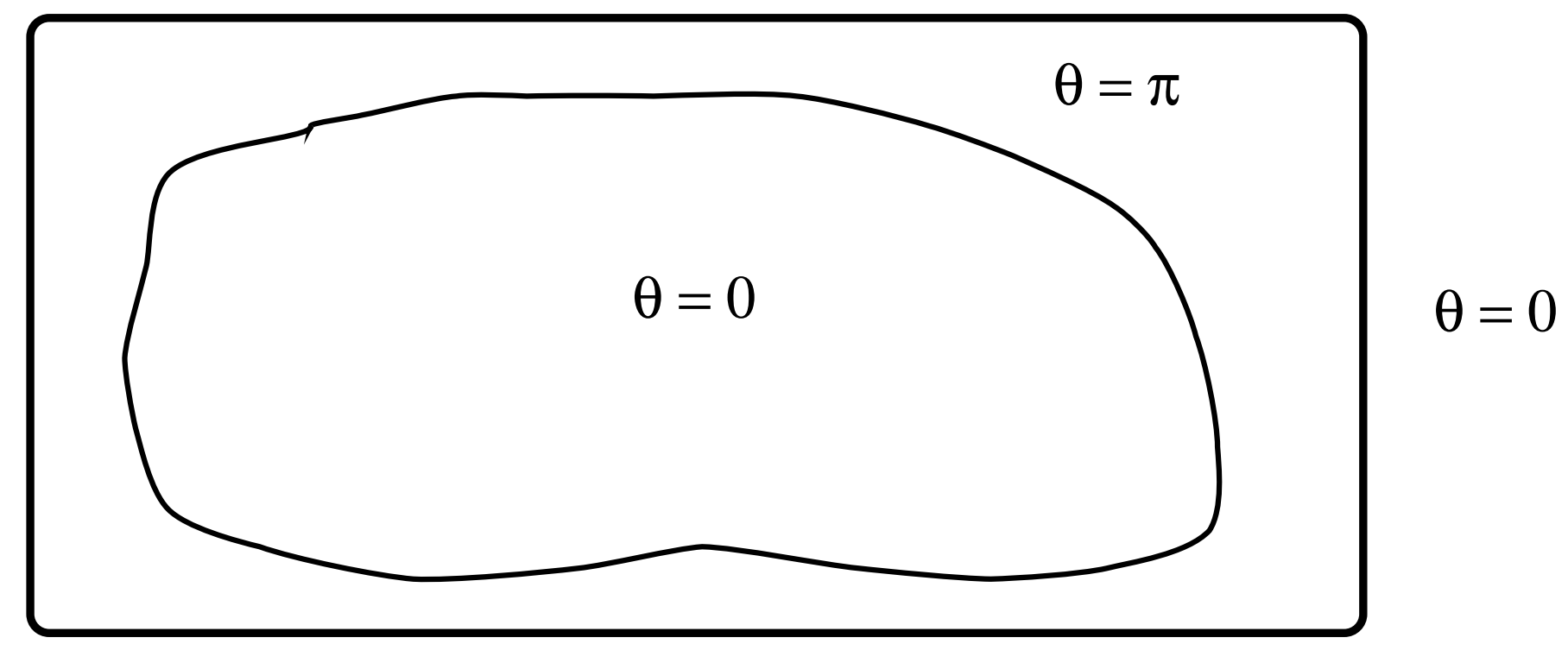

Fig. 6 